\title{
DIVERSITY OF MEDICINAL PLANTS IN ERATTI HILL, THAMARAIKARAI BEAT OF BARGUR RESERVE FOREST, WESTERN GHATS IN ERODE DISTRICT, TAMILNADU, INDIA
}

\author{
VIJAYASHALINI P*, ABIRAMI P \\ PG and Research Department of Botany, Vellalar College for Women, Erode, Tamil Nadu, India. Email: vijayashalinip@gmail.com
}

Received: 27 July 2018, Revised and Accepted: 24 July 2018

\section{ABSTRACT}

Objective: This study was planned to document the medicinal plants in Eratti hill.

Methods: The medicinal plants were collected during their flowering period from July to September. Voucher specimens of all medicinally valuable plants were collected, poisoned, dried, and mounted with voucher number following the conventional methods.

Results: Survey of medicinal plants wealth of Eratti hill, Bargur reserve forest, Western Ghats of Tamil Nadu, India, was carried out during 20162018. 295 medicinal plant species belonging to 66 families were documented. Of these, the highest number of species belongs to the families were Asteraceae, Acanthaceae, Euphorbiaceae, Fabaceae, Convolvulaceae, Solanaceae, Amaranthaceae, Lamiaceae, Asclepiadaceae, Malvaceae, Mimosaceae, and Apocynaceae. Herbs held the dominant position followed by shrubs, trees, climbers, and epiphytes. The medicinal plants were used to treat various ailments such as skin diseases, rheumatoid arthritis, asthma, fever, colic pain, biliousness, wounds eczema, cardiac disorders, and eye diseases used by the Solagars, Lingayats, and Malayali tribals in the hill.

Conclusion: Medicinal plants in Eratti hill play a significant role in primary health care of the ethnic people. This study provides knowledge about herbal treatment of the ethnic people and subsequent pharmacognostical and pharmacological investigations should be made to confirm their mode of preparations.

Keywords: Eratti hill, Medicinal plants, Data collection, Therapeutic uses.

(c) 2018 The Authors. Published by Innovare Academic Sciences Pvt Ltd. This is an open access article under the CC BY license (http://creativecommons. org/licenses/by/4. 0/) DOI: http://dx.doi.org/10.22159/ajpcr.2018.v11i10.27905

\section{INTRODUCTION}

Plants play an important role in traditional medicine, and according to the World Health Organization (WHO), about $65-80 \%$ of the world's population in developing countries depends essentially on plants for their primary health care due to poverty and lack of access to modern medicine [1]. The knowledge of the medicinal plants has been accumulated in the course of many centuries based on different medical systems such as Ayurveda, Unani, and Siddha [2]. India is about $8 \%$ of the estimated biodiversity of the world with around 12,600 species. It is one of the 12 mega biodiversities with two hotspots in the Western Ghats and North-Eastern region [3,4]. It has the rich heritage of using medicinal plants in folklore practices [5]. The traditional people have knowledge of medicinal uses of plants growing around them and used for the treatment of common diseases [6]. The traditional healers of Asia use about 2500 plant species and 100 species of plants serve as regular source of medicine [7]. The knowledge about medicinal plants has been passed orally without any written documents from one generation to other [8]. Ethnomedicinal studies are a suitable source of information regarding useful medicinal plants that can be targeted for domestication and management [9]. These studies are major importance and enhancing the traditional knowledge about medicinal plants. The use of ethnobotanical information in medicinal plant research has gained considerable attention in segment of the scientific community [10], and several active compounds have been discovered from plants on the basis of ethnobotanical information and used directly as patented drugs [11]. Hence, the present study aimed to survey the diversity of medicinal plants and their remedial practices among Lingayats, Solagars, and Malayali tribals of Thamaraikarai beat of Bargur reserve forest, Western Ghats of Erode district. The aboriginal community is familiar with the very intricate rules for collecting plants for use as medicine, such as the time of collection, parts to be collected and care in the conservation and management aspects of medicinal plants. Despite the fact that the area is environmentally degraded, moderate number of medicinal plants categorized in diverse genera and families were recorded. This demonstrates the taxonomic diversity of medicinal plants grown in the Eratti hill as well as the immense knowledge associated with the plants. During the survey, it was also learned that the traditional knowledge regarding medicinal plants and the knowledge is passed down from generation to generation only by means of verbal communication. Therefore, it is necessary to popularize the identity and utility of the medicinal plants.

\section{METHODS}

Study area

Eratti hill of Thamaraikarai beat of Bargur reserve forest is located in the North East of Erode district in Tamil Nadu, India, and the hill is about $70 \mathrm{~km}$ away from Erode. It comes under the part of Western Ghats. The total area of Thamaraikarai beat is about 3066.55 hectares and the Eratti hill is about 600 hectares. The latitude is $11^{\circ} 45.963^{\prime} \mathrm{N}$, the longitude is $077^{\circ} 33.58^{\prime} \mathrm{E}$, and the altitude is $1054 \mathrm{~m}$ above mean sea level. The mean temperature of the hill is around $25^{\circ} \mathrm{C}$ in the winter, and in summer, it is $32^{\circ} \mathrm{C}$ and the annual rainfall of this hill ranges from $400 \mathrm{~mm}$ to $750 \mathrm{~mm}$. The people of the Eratti hill and the surroundings are Lingayats, Solagars, Malayalis, Gounders, and Padayachi. Solagars, Lingayats, and Malayalis are ethnic people inhabiting the hill around 400 years. The main food of the ethnic people is ragi, maize, saamai, and bajra, and the other people used rice as their main food. The main occupation of the people is agriculture and cattle rearing. The people speak Tamil and Kannada. The Eratti hill is surrounded by Thevar malai, Bejilety, Oosi malai, Solaganai, Bodha malai, Kuttaiyur, Onnakarai, Sundappur, Thamratti, Periyasengulam, Sinna sengulam, Koil natham, and Velampatti. A temporary waterfall occurs at the top of the hill and flows in the adjacent areas. There is a dam called Varattupallam at the foothill. The photography of the study area and map was showed in Plate 1a-f. 


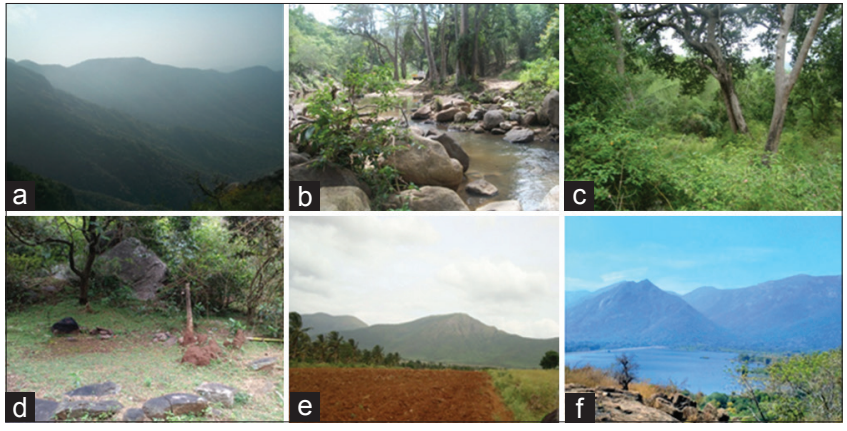

Plate 1: (a) Panoramic view of Eratti hill, (b) A stream of Eratti forest. (c) A dense forest. (d) Unbuilt Sivan temple in forest. (e) Cultivation land of tribals. (f) Varattupallam dam at the foothill

\section{Data collection}

The medicinal plants were collected during their flowering period (July-November). Voucher specimens of all medicinally valuable plants were collected, poisoned, dried, and mounted with voucher number following the conventional methods [12] and deposited at the Department of Botany Herbarium, Vellalar College for Women, Erode, Tamil Nadu. Photographs of few plants were also taken to supplement the herbarium.

\section{Identification}

Identification of medicinal plants was done using Flora of the Presidency of Madras, Gamble and Fischer [13] and the flora of the Tamil Nadu Carnatic, Matthew [14-18]. Flora of Tamil Nadu series [1921] was used at best for nomenclature. The plants were enumerated following the natural system of classification of Bentham and Hooker

Table 1: The list of some medicinally important plants in Eratti hill

\begin{tabular}{|c|c|c|c|c|}
\hline S. No. & Botanical name/family & $\begin{array}{l}\text { Vernacular name/ } \\
\text { common name }\end{array}$ & Parts used & Therapeutic uses \\
\hline 1. & $\begin{array}{l}\text { Clematis gouriana Roxb. } \\
\text { Ranunculaceae }\end{array}$ & - & $\begin{array}{l}\text { Whole plant and } \\
\text { leaves paste }\end{array}$ & $\begin{array}{l}\text { Whole plant juice - cold, headache, wound } \\
\text { healing. Leaf extract applied externally for } \\
\text { eczema, boils, and itches, leaf paste applied to } \\
\text { scabies, cuts, and wounds }\end{array}$ \\
\hline 2. & $\begin{array}{l}\text { Annona reticulata } \\
\text { L. Annonaceae }\end{array}$ & Ramchita & $\begin{array}{l}\text { Fruit, leaves, whole } \\
\text { plant }\end{array}$ & $\begin{array}{l}\text { To treat dysentery, epilepsy, cardiac problem, } \\
\text { constipation, hemorrhage, bacterial } \\
\text { infection, fever, ulcer and the plants have } \\
\text { anti-inflammatory, antipyretic, anthelmintic, } \\
\text { cytotoxic effects, analgesic and wound healing } \\
\text { properties }\end{array}$ \\
\hline 3. & $\begin{array}{l}\text { Cissampelos pareira } \\
\text { L. Menispermaceae }\end{array}$ & Malai mattiri & Roots, leaves & $\begin{array}{l}\text { The roots astringent, anthelminthic, } \\
\text { carminative, stomachin, digestive, anti- } \\
\text { inflammatory, pungent. Diuretic, febrifuge, } \\
\text { expectorant, galactagogue, diuretic, febrifuge, } \\
\text { expectorant, galactagogue, and bitter tonic. It } \\
\text { is used in dyspepsia, indigestion, flatulence, } \\
\text { abdominal pains, diarrhea, dysentery, blood } \\
\text { disorders, cardiac disorders, edema, leprosy, } \\
\text { sensation, cough, coryza, asthma, bronchitis, } \\
\text { cystitis, dysuria, and lactation disorders }\end{array}$ \\
\hline 4. & $\begin{array}{l}\text { Nymphaea stellata Willd. } \\
\text { Nymphaeaceae }\end{array}$ & Alli & Whole plant & $\begin{array}{l}\text { Whole plant - liver disorders in Ayurveda. } \\
\text { Leaves, roots, and flowers - diabetes, } \\
\text { antifertility, heart troubles, dysentery, eruptive } \\
\text { fevers, indigestion and as a cardiotonic, } \\
\text { emollient, diuretic, narcotics, stimulant, and } \\
\text { aphrodisiac }\end{array}$ \\
\hline 5. & $\begin{array}{l}\text { Argemone mexicana } \\
\text { L. Papaveraceae }\end{array}$ & Brammathandu & $\begin{array}{l}\text { Leaves, roots, and } \\
\text { whole plant }\end{array}$ & $\begin{array}{l}\text { Leaves - malarial fever, ulcers, and skin } \\
\text { problems. } \\
\text { Root - chronic skin diseases. } \\
\text { Plant juice - jaundice and skin problems. } \\
\text { Latex - cataract, reddening, and itching in the } \\
\text { eyes }\end{array}$ \\
\hline 6. & Cleome monophylla L. Cleomaceae & $\begin{array}{l}\text { Ellukku sakkalathi } \\
\text { and Naai kaduku }\end{array}$ & Whole plant & Anti-HIV activity \\
\hline 7. & Cleome felina L.f. Cleomaceae & Cuvarnaciri Taivelai & Whole plant & $\begin{array}{l}\text { Anticancer, anti-inflammatory, antimicrobial } \\
\text { activities }\end{array}$ \\
\hline 8. & $\begin{array}{l}\text { Capparis divaricata Lam. } \\
\text { Capparidaceae }\end{array}$ & Thoratti & $\begin{array}{l}\text { Whole plant, roots, } \\
\text { and leaves }\end{array}$ & $\begin{array}{l}\text { Whole plant - blood purifier stomachic, tonic, } \\
\text { and appetizer } \\
\text { Root powder - inflammation, muscles diseases, } \\
\text { and snakebites }\end{array}$ \\
\hline 9. & Capparis grandis L.f. Capparidaceae & & Whole plant & $\begin{array}{l}\text { Inflammations, skin, renal, and central nervous } \\
\text { disorders }\end{array}$ \\
\hline 10. & $\begin{array}{l}\text { Ionidium suffruticosum Ging. } \\
\text { Violaceae }\end{array}$ & Orithal thamarai & $\begin{array}{l}\text { Whole plant, roots, } \\
\text { and leaves }\end{array}$ & $\begin{array}{l}\text { Whole plant - tonic, demulcent and diuretic, } \\
\text { strangury and to treat dysentery, vomiting, } \\
\text { asthma, and cough } \\
\text { Root - diuretic, gonorrhea, and urinary affections } \\
\text { Dried powdered leaves-asthma }\end{array}$ \\
\hline
\end{tabular}


Table 1: (Continued)

\begin{tabular}{|c|c|c|c|c|}
\hline S. No. & Botanical name/family & $\begin{array}{l}\text { Vernacular name/ } \\
\text { common name }\end{array}$ & Parts used & Therapeutic uses \\
\hline 11. & $\begin{array}{l}\text { Polygala javana DC. } \\
\text { Polygalaceae }\end{array}$ & - & Whole plant & Asthma \\
\hline 12. & $\begin{array}{l}\text { Shorea roxburghii G.Don. } \\
\text { Dipterocarpaceae }\end{array}$ & Talari & Resins & Headache and chest pain in children \\
\hline 13. & $\begin{array}{l}\text { Malvastrum coromandelianum Garcke. } \\
\text { Malvaceae }\end{array}$ & - & Whole plant & $\begin{array}{l}\text { Whole plant - dysentery, stomach pain, and } \\
\text { wounds }\end{array}$ \\
\hline 14. & $\begin{array}{l}\text { Sida acuta Burm. } \\
\text { Malvaceae }\end{array}$ & Ariva-mooku keerai & Whole plant, leaves & $\begin{array}{l}\text { Plant decoction - fever, dysentery, digestive } \\
\text { disorders, and wounds } \\
\text { Leaves - headache, ulcers, and throats }\end{array}$ \\
\hline 15. & $\begin{array}{l}\text { Pavonia odorata Willd. } \\
\text { Malvaceae }\end{array}$ & Peramutti & Root powder & Skin disorders \\
\hline 16. & $\begin{array}{l}\text { Pavonia zeylanica Cav. } \\
\text { Malvaceae }\end{array}$ & Sevagan & Leaves paste & $\begin{array}{l}\text { Applied externally once a day for } 3 \text { days to cure } \\
\text { swellings }\end{array}$ \\
\hline 17. & $\begin{array}{l}\text { Hibiscus syriacus L. } \\
\text { Malvaceae }\end{array}$ & - & Whole plant & $\begin{array}{l}\text { Inflammation, cough, cold, skin diseases, and } \\
\text { urinary tract diseases }\end{array}$ \\
\hline 18. & Helicteres isora L. Sterculiaceae & Valampuri & $\begin{array}{l}\text { Leaf, fruit, and seed } \\
\text { decoctions }\end{array}$ & $\begin{array}{l}\text { Leaves - skin diseases } \\
\text { Fruit - diarrhea, asthma, and cough } \\
\text { Seed decoction - abdominal diseases }\end{array}$ \\
\hline 19. & $\begin{array}{l}\text { Melhania incana Heyne } \\
\text { Sterculiaceae }\end{array}$ & - & Whole plant & Fever, cold, and cough \\
\hline 20. & $\begin{array}{l}\text { Grewia tiliaefolia Vahl. } \\
\text { Tiliaceae }\end{array}$ & Palisamaram & $\begin{array}{l}\text { Stem and root bark } \\
\text { decoctions }\end{array}$ & $\begin{array}{l}\text { Stem bark decoction - to improve the hair } \\
\text { growth and will reduce pittham } \\
\text { Root bark decoction - bone fracture of animals and } \\
\text { possesses analgesic and anti-inflammatory activities }\end{array}$ \\
\hline 21. & $\begin{array}{l}\text { Triumfetta pentandra A.Rich. } \\
\text { Tiliaceae }\end{array}$ & Kapodam & Roots & $\begin{array}{l}\text { Root extract is mixed with sugar candy and } \\
\text { one cup of this mixture is taken during early } \\
\text { morning for } 6 \text { days to increase sperm count }\end{array}$ \\
\hline 22. & $\begin{array}{l}\text { Erythroxylum monogynum Roxb. } \\
\text { Erythroxylaceae }\end{array}$ & Devadara & Leaves & Malaria, jaundice, and kill intestinal worms \\
\hline 23. & $\begin{array}{l}\text { Oxalis latifolia Kunth. } \\
\text { Oxalidaceae }\end{array}$ & Tenpaku & Leaves & To cure acidity \\
\hline 24. & $\begin{array}{l}\text { Toddalia asiatica Lam. } \\
\text { Rutaceae }\end{array}$ & Kaatu milagu & Leaves, fruit, root & $\begin{array}{l}\text { Leaves - rheumatism, lung diseases stomach } \\
\text { ache, and snakebites, fruit - cough and malaria } \\
\text { Roots to treat influenza and indigestion }\end{array}$ \\
\hline 25. & $\begin{array}{l}\text { Chloroxylon swietenia DC. } \\
\text { Rutaceae }\end{array}$ & Porasu & $\begin{array}{l}\text { Whole plant and } \\
\text { stem bark }\end{array}$ & Anemia, cough, rheumatism, and jaundice \\
\hline 26. & Murraya exotica L. Rutaceae & Kaatu kariveppilai & Leaf extract & Diarrhea and inflammations \\
\hline 27. & $\begin{array}{l}\text { Ailanthus malabarica DC. } \\
\text { Simaroubaceae }\end{array}$ & Peru & $\begin{array}{l}\text { Whole plant and } \\
\text { stem }\end{array}$ & $\begin{array}{l}\text { Whole plant - dyspepsia, bronchitis, ophthalmic, } \\
\text { and snakebite } \\
\text { Dried stem bark - dysentery and diarrhea }\end{array}$ \\
\hline 28. & $\begin{array}{l}\text { Cipadessa baccifera Miq. } \\
\text { Meliaceae }\end{array}$ & Pullip panchedi & Leaf juice and paste & $\begin{array}{l}\text { Hand and leg pains } \\
\text { Leaf paste - applied externally to cure piles. }\end{array}$ \\
\hline 29. & $\begin{array}{l}\text { Maytenus heyneana (Roth) D.C.S.Raju. } \\
\text { and Babu. } \\
\text { Celastraceae }\end{array}$ & - & Whole plant & Antitumor activity \\
\hline 30. & $\begin{array}{l}\text { Zizyphus nummularia W. and A. } \\
\text { Rhamnaceae }\end{array}$ & Sooraipalam & Leaves and fruits & $\begin{array}{l}\text { The leaves - skin problems and scabies } \\
\text { Dried fruits - astringent }\end{array}$ \\
\hline 31. & $\begin{array}{l}\text { Ziziphus glabrata W. } \\
\text { Rhamnaceae }\end{array}$ & Kaatu elanthai & Flowers & Menorrhagia \\
\hline 32. & $\begin{array}{l}\text { Cayratia pedata Juss. } \\
\text { Vitaceae }\end{array}$ & Kaatupirantai & $\begin{array}{l}\text { Aerial parts and } \\
\text { leaves }\end{array}$ & $\begin{array}{l}\text { Asthma, coughs, bronchitis, and joint pain } \\
\text { Leaves - antiarthritis activity }\end{array}$ \\
\hline 33. & $\begin{array}{l}\text { Cardiospermum canescens Wall. } \\
\text { Sapindaceae }\end{array}$ & Kaatu mudakkathn & Whole plant & Dysentery and rheumatoid arthritis \\
\hline 34. & $\begin{array}{l}\text { Filicium decipiens Thw. } \\
\text { Sapindaceae }\end{array}$ & Ningal & Barks & Bark decoction - body pain and headache \\
\hline 35. & $\begin{array}{l}\text { Dodonaea angustifolia L.f. } \\
\text { Sapindaceae }\end{array}$ & Virali & Leaves and barks & $\begin{array}{l}\text { Leaves and barks are grounded and applied for } \\
\text { bone displacement and muscular stiffness }\end{array}$ \\
\hline 36 & $\begin{array}{l}\text { Buchanania lanzan Spr. } \\
\text { Anacardiaceae }\end{array}$ & Charam & $\begin{array}{l}\text { Whole plant and } \\
\text { stem bark paste }\end{array}$ & $\begin{array}{l}\text { The whole plant - taken orally for diarrhea } \\
\text { Stem bark paste - antidote for snakebite }\end{array}$ \\
\hline 37. & $\begin{array}{l}\text { Odina wodier Roxb. } \\
\text { Anacardiaceae }\end{array}$ & Otti & Whole plant & The plants possess anti-inflammatory activities \\
\hline 38. & $\begin{array}{l}\text { Crotalaria calycina Schr. } \\
\text { Fabaceae }\end{array}$ & - & Whole plant & $\begin{array}{l}\text { Wounds, convulsions, venereal sores, cholera, } \\
\text { hematuria, dysentery, and syphilis }\end{array}$ \\
\hline 39. & Crotalaria verrucosa L. Fabaceae & Sangunithi & Leaves & $\begin{array}{l}\text { The leaves - emetic, expectorant, biliousness, } \\
\text { dyspepsia, heart complaints, fever, and mouth diseases }\end{array}$ \\
\hline
\end{tabular}


Table 1: (Continued)

\begin{tabular}{|c|c|c|c|c|}
\hline S. No. & Botanical name/family & $\begin{array}{l}\text { Vernacular name/ } \\
\text { common name }\end{array}$ & Parts used & Therapeutic uses \\
\hline 40. & $\begin{array}{l}\text { Crotalaria pallida Aiton } \\
\text { Fabaceae }\end{array}$ & Kilukillupai chedi & $\begin{array}{l}\text { Whole plant, roots, } \\
\text { and leaf extracts }\end{array}$ & $\begin{array}{l}\text { Urinary problem, fever, prevents skin infections, } \\
\text { and eczema } \\
\text { Roots - cure painful swelling of joint } \\
\text { Leaf extracts act as vermifuge }\end{array}$ \\
\hline 41. & Indigofera enneaphylla L. Fabaceae & Seppu nerunji & Whole plant & Wound healing properties \\
\hline 42. & $\begin{array}{l}\text { Desmodium gangeticum DC. } \\
\text { Fabaceae }\end{array}$ & Pulladi & Root & $\begin{array}{l}\text { Roots - digestive, laxative, diuretic, anti- } \\
\text { inflammatory, and anticatarrhal activity and to treat } \\
\text { cough, diarrhea, biliousness, emetic, piles, asthma, } \\
\text { rheumatism, arthritis, and post-delivery problems }\end{array}$ \\
\hline 43. & $\begin{array}{l}\text { Butea monosperma (Lam.) Taub. } \\
\text { Fabaceae }\end{array}$ & Kinchugam or parasu & Leaves and seeds & $\begin{array}{l}\text { Leaves - insects bites } \\
\text { Seeds have purgative, diuretic, and anthelmintic }\end{array}$ \\
\hline 44. & $\begin{array}{l}\text { Pterocarpus santalinus L.f. } \\
\text { Fabaceae }\end{array}$ & Sivappu santhanam & Wood oil and paste & $\begin{array}{l}\text { Wood oil - spider bite } \\
\text { Wood paste - removes warts }\end{array}$ \\
\hline 45. & Cassia hirsuta L. Caesalpiniaceae & - & $\begin{array}{l}\text { Leaf decoction, } \\
\text { paste, and root }\end{array}$ & $\begin{array}{l}\text { Cure kidney stone and the leaf paste - applied } \\
\text { externally to cure skin problems } \\
\text { Roots - rheumatic pain }\end{array}$ \\
\hline 46. & $\begin{array}{l}\text { Acacia modesta Wall. } \\
\text { Mimosaceae }\end{array}$ & - & Whole plant & Leukorrhea, back pain, and sexual debility \\
\hline 47. & Acacia nilotica L. Mimosaceae & Karuvelam & $\begin{array}{l}\text { Leaves, fruits, seeds, } \\
\text { stem bark, gum, and } \\
\text { roots. }\end{array}$ & $\begin{array}{l}\text { Anticancer, antipyretic, antiasthmatic, } \\
\text { antidiabetic, antiplasmodial, and antifungal } \\
\text { activities }\end{array}$ \\
\hline 48. & $\begin{array}{l}\text { Pterolobium indicum A.Rich. } \\
\text { Mimosaceae }\end{array}$ & Indumullu & Young leaves & $\begin{array}{l}\text { Diarrhea, jaundice, skin infections, piles, ulcers, } \\
\text { constipation, wound healing, and coughs }\end{array}$ \\
\hline 49. & $\begin{array}{l}\text { Rubus ellipticus Sm. } \\
\text { Rosaceae }\end{array}$ & Mullippazham. & Roots and leaves & Stomach disorders \\
\hline 50. & $\begin{array}{l}\text { Drosera spatulata Labill. } \\
\text { Droseraceae }\end{array}$ & Alugini cedi & Whole plant & Asthma, lung diseases, and ulcers \\
\hline 51. & $\begin{array}{l}\text { Anogeissus latifolia (Roxb. ex DC.) } \\
\text { Wall. ex Guill. \& Perr. } \\
\text { Combretaceae }\end{array}$ & Vel-naga-maram & Stem bark infusion & Stem bark infusion \\
\hline 52. & $\begin{array}{l}\text { Syzigium aromaticum (L) Merr. and } \\
\text { Perry. } \\
\text { Myrtaceae }\end{array}$ & Kirambu & Whole plant & Anti-inflammatory and analgesic properties \\
\hline 53. & Ammannia baccifera L. Lythraceae & Kalluruvi & $\begin{array}{l}\text { Leaves and whole } \\
\text { plant }\end{array}$ & $\begin{array}{l}\text { Plant extracts possess antibacterial properties. } \\
\text { Used as laxative, stomachic, strangury, cure } \\
\text { biliousness (stomach problems), ulcers, and } \\
\text { rheumatic pain }\end{array}$ \\
\hline 54. & $\begin{array}{l}\text { Passiflora edulis Sims } \\
\text { Passifloraceae }\end{array}$ & - & Leaves & To control blood pressure \\
\hline 55. & Bryonia laciniosa L. Cucurbitaceae & Sivalingi & Whole plant & $\begin{array}{l}\text { Used as an antibacterial, antifungal, anti- } \\
\text { inflammatory, cytotoxic, analgesic, and } \\
\text { antipyretic agent }\end{array}$ \\
\hline 56. & $\begin{array}{l}\text { Citrullus colocynthis Schrad. } \\
\text { Cucurbitaceae }\end{array}$ & Varikura mathai & Whole plant & $\begin{array}{l}\text { Plant possesses antioxidant, antidiabetic, anti- } \\
\text { inflammatory, antimicrobial, gastrointestinal, } \\
\text { anticancerm, and analgesic properties }\end{array}$ \\
\hline 57. & $\begin{array}{l}\text { Centella asiatica Urb. } \\
\text { Apiaceae }\end{array}$ & Vallarai & Leaves and stem & $\begin{array}{l}\text { It improves the memory power and blood } \\
\text { circulations, stimulates the hair growth, tissue } \\
\text { strengthens, reducing arthritis, and wound healing }\end{array}$ \\
\hline 58. & $\begin{array}{l}\text { Canthium dicoccum (Gaertn.) Merr. } \\
\text { Rubiaceae }\end{array}$ & Nanjul & Whole plant & $\begin{array}{l}\text { The plants possess antimicrobial, antitumor, and } \\
\text { antioxidant properties }\end{array}$ \\
\hline 59. & $\begin{array}{l}\text { Mitragyna parvifolia Korth. } \\
\text { Rubiaceae }\end{array}$ & Neer kadambu & Stem bark paste & To relief rheumatic pain \\
\hline 60. & Richardia scabra L. Rubiaceae & - & Whole plant & $\begin{array}{l}\text { Skin diseases, urinary tract infections, asthma, } \\
\text { emetic, and dermatitis }\end{array}$ \\
\hline 61. & Rubia cordifolia L. Rubiaceae & Manjitti & Roots & $\begin{array}{l}\text { Bronchitis, kidney stones, dysentery, blood } \\
\text { pressure, and rheumatism }\end{array}$ \\
\hline 62. & $\begin{array}{l}\text { Vernonia cinerea Less. } \\
\text { Asteraceae }\end{array}$ & Neichattipoo & $\begin{array}{l}\text { Whole plant, leaf, } \\
\text { and stem bark paste }\end{array}$ & $\begin{array}{l}\text { Whole plant - inflammation, arthritis, flatulence } \\
\text { Leaves - tonsillitis, conjunctivitis, leukoderma } \\
\text { Stem bark paste - to treat piles }\end{array}$ \\
\hline 63. & $\begin{array}{l}\text { Vicoa indica DC. } \\
\text { Asteraceae }\end{array}$ & Mookuthi poo & Leaf decoction & Gastric troubles \\
\hline 64. & Siegesbeckia orientalis L. Asteraceae & Kathambam & $\begin{array}{l}\text { Plant juice, } \\
\text { decoction, and root } \\
\text { paste }\end{array}$ & $\begin{array}{l}\text { Plant juice - applied for externally to treat } \\
\text { wounds and parasitic infections } \\
\text { Plant decoction - rheumatoid arthritis } \\
\text { Root paste - wounds }\end{array}$ \\
\hline
\end{tabular}


Table 1: (Continued)

\begin{tabular}{|c|c|c|c|c|}
\hline S. No. & Botanical name/family & $\begin{array}{l}\text { Vernacular name/ } \\
\text { common name }\end{array}$ & Parts used & Therapeutic uses \\
\hline 65. & Senecio vulgaris L. Asteraceae & - & Whole plant & $\begin{array}{l}\text { Antiscorbutic, diaphoretic, anthelmintic, } \\
\text { purgative, and diuretic }\end{array}$ \\
\hline 66. & $\begin{array}{l}\text { Plumbago zeylanica L. } \\
\text { Plumbaginaceae }\end{array}$ & Chithira moolam & Whole plant & Diabetes skin wounds, rashes \\
\hline 67. & Rauvolfia tetraphylla L. Apocynaceae & Pampukaalaachchedi & Roots & $\begin{array}{l}\text { To cure mental disorders and high blood } \\
\text { pressure }\end{array}$ \\
\hline 69. & $\begin{array}{l}\text { Gomphocarpus physocarpus E.Mey } \\
\text { Apocynaceae }\end{array}$ & - & Latex & To treat wounds \\
\hline 70. & $\begin{array}{l}\text { Oxystelma esculentum R.Br. } \\
\text { Asclepiadaceae }\end{array}$ & Anaitta vitakkoti & $\begin{array}{l}\text { Whole plant, leaves, } \\
\text { and fruits }\end{array}$ & $\begin{array}{l}\text { Ulcer, itches, and sore throats } \\
\text { Crushed leaves - jaundice and fruits - body pain } \\
\text { and cold }\end{array}$ \\
\hline 71. & $\begin{array}{l}\text { Sarcostemma brevistigma W. and A. } \\
\text { Asclepiadaceae }\end{array}$ & Kodikalli & Whole plant & $\begin{array}{l}\text { To cure emetic } \\
\text { Plants possess antimicrobial activities }\end{array}$ \\
\hline 72. & $\begin{array}{l}\text { Wattakka volubilis (L. fil.) Stapf. } \\
\text { Asclepiadaceae }\end{array}$ & Kurincha & $\begin{array}{l}\text { Whole plant and } \\
\text { roots }\end{array}$ & $\begin{array}{l}\text { Piles, tumors, leukoderma, asthma, eye diseases, } \\
\text { cold } \\
\text { Roots are used as an expectorant }\end{array}$ \\
\hline 73. & $\begin{array}{l}\text { Exacum pedunculatum L. } \\
\text { Gentianaceae }\end{array}$ & Kanapoondu & Whole plant & $\begin{array}{l}\text { Antimicrobial activities and to treat stomach } \\
\text { ache and fever }\end{array}$ \\
\hline 74. & Anagallis arvensis L. Gentianaceae & - & Dried plant powder & Leprosy, gout, and hydrophobia \\
\hline 75. & Cordia oblique Wild. Boraginaceae & Naruvili & Fruit & Cough, the disease of chest, and chronic fever \\
\hline 76. & $\begin{array}{l}\text { Rivea hypocrateriformis Choisy. } \\
\text { Convolvulaceae }\end{array}$ & Musuttai & Whole plant & Antioxidant property \\
\hline 77. & $\begin{array}{l}\text { Ipomoea obscura K.Gawl. } \\
\text { Convolvulaceae }\end{array}$ & Chirutali & Leaf paste & Ulcers, swellings, and hemorrhoids \\
\hline 78. & $\begin{array}{l}\text { Ipomoea pes-tigridis } \\
\text { L. Convolvulaceae }\end{array}$ & Pulichovadi & Whole plant & $\begin{array}{l}\text { To treat headaches, swelling, pain, and } \\
\text { snakebites }\end{array}$ \\
\hline 79. & $\begin{array}{l}\text { Evolvulus nummularius } \\
\text { L. Convolvulaceae }\end{array}$ & Elikkathu illai & Leaves & Purify blood and improve memory power \\
\hline 80. & $\begin{array}{l}\text { Solanum surattense Burm.f. } \\
\text { Solanaceae }\end{array}$ & Kandan kathiri & Whole plant & $\begin{array}{l}\text { Skin diseases, cough, bronchitis, anorexia, and } \\
\text { urinary tract disorders }\end{array}$ \\
\hline 82. & $\begin{array}{l}\text { Datura discolor Bernh. } \\
\text { Solanaceae }\end{array}$ & Karu oomathai & Leaves & $\begin{array}{l}\text { Leaves - pain, asthma, and bone setting } \\
\text { Seeds are antispasmodic, hypnotic, and narcotic }\end{array}$ \\
\hline 83. & Scoparia dulcis L. Scrophulariaceae & Kallurukki & Grounded leaves & Piles \\
\hline 84. & $\begin{array}{l}\text { Thunbergia fragrans Roxb. } \\
\text { Acanthaceae }\end{array}$ & Indra pushpam & Root decoctions & $\begin{array}{l}\text { Used as a health tonic } \\
\text { Plants have an antioxidant, hepatoprotective, } \\
\text { anthelmintic, and antibacterial activities }\end{array}$ \\
\hline 85. & Ruellia tuberosa L. Acanthaceae & Kiranthi nayagam & Root & Kidney stones and whooping coughs \\
\hline 86. & $\begin{array}{l}\text { Andrographis echioides Nees. } \\
\text { Acanthaceae }\end{array}$ & Koopuram tangi & Leaf juice & To promote hair growth \\
\hline 87. & $\begin{array}{l}\text { Rhinacanthus nasutus (L.) Kurz. } \\
\text { Acanthaceae }\end{array}$ & Nagamalli & Whole plant & $\begin{array}{l}\text { Blood pressure, allergy, skin diseases, and } \\
\text { inflammation, prevent cancer, and diabetes }\end{array}$ \\
\hline 88. & $\begin{array}{l}\text { Peristrophe paniculata (Forssk.) R.k. } \\
\text { Acanthaceae }\end{array}$ & Nagananda & Whole plant & Tuberculosis \\
\hline 89. & Gmelina asiatica L. Verbenaceae & Mulkumizh & $\begin{array}{l}\text { Grounded leaves } \\
\text { extract }\end{array}$ & Fever \\
\hline 90. & Ocimum gratissimum L. Lamiaceae & Ramthulasi & Whole plant & Rheumatism and cough \\
\hline 91. & $\begin{array}{l}\text { Coleus barbatus Benth. } \\
\text { Lamiaceae }\end{array}$ & Kattu valli & Whole plant & Bronchitis, asthma, cold, cough, and sore throat \\
\hline 92. & $\begin{array}{l}\text { Anisochilus carnosus Wall. } \\
\text { Lamiaceae }\end{array}$ & Kanakavalli cedi & $\begin{array}{l}\text { Grounded and } \\
\text { boiled leaves and } \\
\text { root piece }\end{array}$ & $\begin{array}{l}\text { Applied externally to cure skin itches } \\
\text { Root piece is chewed to cure cold }\end{array}$ \\
\hline 93. & $\begin{array}{l}\text { Anisomeles indica } 0 . \text { Kze. } \\
\text { Lamiaceae }\end{array}$ & Sirumiratti & Whole plant & $\begin{array}{l}\text { Rheumatism, cold, fever, skin sores, and } \\
\text { abdominal pain }\end{array}$ \\
\hline 94. & $\begin{array}{l}\text { Scutellaria violacea Heyne } \\
\text { Lamiaceae }\end{array}$ & Novu pacchilai & Whole plant & $\begin{array}{l}\text { Chronic, fatigue, skin diseases, and urinary } \\
\text { infection }\end{array}$ \\
\hline 95. & $\begin{array}{l}\text { Leucas longifolia } \text { Hk. f. } \\
\text { Lamiaceae }\end{array}$ & Kaatu thumbai & Whole plant & Antimicrobial activity \\
\hline 96. & $\begin{array}{l}\text { Allmania longepedunculata Gamble } \\
\text { n. Comb. } \\
\text { Amaranthaceae }\end{array}$ & Kumutti & Whole plant & Cough, malaria, and hair fall \\
\hline 97. & $\begin{array}{l}\text { Pupalia lappacea Moq. } \\
\text { Amaranthaceae }\end{array}$ & - & Whole plant & $\begin{array}{l}\text { Antioxidant, wound healing, and antibacterial } \\
\text { activities }\end{array}$ \\
\hline
\end{tabular}


Table 1: (Continued)

\begin{tabular}{|c|c|c|c|c|}
\hline S. No. & Botanical name/family & $\begin{array}{l}\text { Vernacular name/ } \\
\text { common name }\end{array}$ & Parts used & Therapeutic uses \\
\hline 98. & $\begin{array}{l}\text { Alternanthera tenella Colla. } \\
\text { Amaranthaceae }\end{array}$ & Ottara mull & Whole plant & Anti-inflammatory activities \\
\hline 99. & $\begin{array}{l}\text { Chenopodium ambrosioides } \mathrm{L} \text {. } \\
\text { Chenopodiaceae }\end{array}$ & Sakkaravarthi keerai & $\begin{array}{l}\text { Whole plant } \\
\text { decoction }\end{array}$ & $\begin{array}{l}\text { Stomach pain, cough, asthma, fever, and } \\
\text { headache }\end{array}$ \\
\hline 100. & $\begin{array}{l}\text { Persicaria chinensis (L.) } \\
\text { Polygonaceae }\end{array}$ & - & Whole plant & $\begin{array}{l}\text { The infusion of the plant is to treat eye diseases } \\
\text { and eczema of the ear }\end{array}$ \\
\hline 101. & $\begin{array}{l}\text { Polygonum persicaria (L.) Small. } \\
\text { Polygonaceae }\end{array}$ & - & Whole plant & $\begin{array}{l}\text { Skin diseases and vaginal diseases } \\
\text { Plant possesses antifungal properties }\end{array}$ \\
\hline 102. & $\begin{array}{l}\text { Aristolochia bracteata Retz. } \\
\text { Aristolochiaceae }\end{array}$ & Aadutheendaappaalai & $\begin{array}{l}\text { Whole plant and leaf } \\
\text { paste }\end{array}$ & $\begin{array}{l}\text { Whole plant - snakebite and rheumatism } \\
\text { Leaves paste - applied externally for treat } \\
\text { wounds and cuts }\end{array}$ \\
\hline 103. & Aristolochia indica L. Aristolochiaceae & Isvaramuli & Root paste & $\begin{array}{l}\text { Given orally to treat snake poison } \\
\text { The root paste with Calotropis gigantea leaves is } \\
\text { externally applied for dog bite }\end{array}$ \\
\hline 104. & $\begin{array}{l}\text { Neolitsea scrobiculata Gamble } \\
\text { Lauraceae }\end{array}$ & - & Whole plant & Antimicrobial activities \\
\hline 105. & $\begin{array}{l}\text { Helixanthera parasitica Lour. } \\
\text { Loranthaceae }\end{array}$ & - & Whole plant & Antioxidant and antimetastatic effect \\
\hline 106. & Santalum album L. Santalaceae & Santhana maram & Wood oil & $\begin{array}{l}\text { Skin disorder, facial warts, and pimples used as } \\
\text { refrigerant }\end{array}$ \\
\hline 107. & $\begin{array}{l}\text { Osyris wightiana Wall. } \\
\text { Santalaceae }\end{array}$ & Vella santhanam & Bark infusion & Given to women after delivery to stop bleeding \\
\hline 108. & $\begin{array}{l}\text { Euphorbia antiquorum } \\
\text { L. Euphorbiaceae }\end{array}$ & Sathurakalli & Latex and stem juice & $\begin{array}{l}\text { The latex applied externally to treat boils, } \\
\text { wounds, rheumatism, and toothache. Stem juice } \\
\text { - earache }\end{array}$ \\
\hline 109. & $\begin{array}{l}\text { Synadenium grantii Hook.f. } \\
\text { Euphorbiaceae }\end{array}$ & - & Whole plant & $\begin{array}{l}\text { Neoplastic and gastric disorders. Plants have } \\
\text { antiulcer and antitumor activity }\end{array}$ \\
\hline 110. & $\begin{array}{l}\text { Phyllanthus maderaspatensis } \\
\text { L. Euphorbiaceae }\end{array}$ & Arunelli & Leaves & $\begin{array}{l}\text { Cough, strangury, and sweating, seeds are } \\
\text { laxative, diuretic, and diaphoretic properties }\end{array}$ \\
\hline 111. & Phyllanthus emblica L. Euphorbiaceae & Malai nelli & Whole plant & Jaundice, inflammation, and diarrhea \\
\hline 112. & $\begin{array}{l}\text { Phyllanthus virgatus G.Forst. } \\
\text { Euphorbiaceae }\end{array}$ & Naduvanelli & Whole plant & $\begin{array}{l}\text { Skin itches, gonorrhea, measles, venereal } \\
\text { diseases, and jaundice }\end{array}$ \\
\hline 113. & $\begin{array}{l}\text { Glochidion zeylanicum A. Juss. } \\
\text { Euphorbiaceae }\end{array}$ & - & Whole plant & The plants have anticancer activity \\
\hline 114 & $\begin{array}{l}\text { Mallotus philippinensis M. Arg. } \\
\text { Euphorbiaceae }\end{array}$ & Kunkuma maram & Whole plant & $\begin{array}{l}\text { Antifilarial, anti-inflammatory and } \\
\text { immunoregulatory, antioxidant, antiradical, } \\
\text { hepatoprotective, and purgative activities }\end{array}$ \\
\hline 115. & $\begin{array}{l}\text { Acalypha fruticosa Forsk. } \\
\text { Euphorbiaceae }\end{array}$ & Sinni & Whole plant & $\begin{array}{l}\text { Dyspepsia, skin disorders, wounds, and } \\
\text { poisonous bites }\end{array}$ \\
\hline 116. & $\begin{array}{l}\text { Acalypha paniculata Wall. } \\
\text { Euphorbiaceae }\end{array}$ & Kozipoondu & Whole plant & Antioxidant properties \\
\hline 117. & Tragia involucrata L. Euphorbiaceae & Kaanjori & Roots and leaves & $\begin{array}{l}\text { Itching skin, bronchitis } \\
\text { Leaves - jaundice }\end{array}$ \\
\hline 118. & Jatropha curcas L. Euphorbiaceae & Kaatukottai & Leaves and seeds & $\begin{array}{l}\text { Leaves - rheumatism and tumor } \\
\text { Seeds - the oil is to treat skin diseases and } \\
\text { rashes }\end{array}$ \\
\hline 119. & $\begin{array}{l}\text { Jatropha glandulifera Roxb. } \\
\text { Euphorbiaceae }\end{array}$ & Vellai kattukottai & $\begin{array}{l}\text { Whole plant oil and } \\
\text { latex }\end{array}$ & $\begin{array}{l}\text { Plant oil - antiparalytic and antirheumatic } \\
\text { properties } \\
\text { Latex applied externally for warts and tumor }\end{array}$ \\
\hline 120. & $\begin{array}{l}\text { Trema orientalis } \mathrm{Bl} . \\
\text { Ulmaceae }\end{array}$ & Ambaratthi & $\begin{array}{l}\text { Root decoction and } \\
\text { stem bark }\end{array}$ & $\begin{array}{l}\text { Root decoction - hematuria, diarrhea, and } \\
\text { epilepsy } \\
\text { Stem bark - muscular pains }\end{array}$ \\
\hline 121. & $\begin{array}{l}\text { Artocarpus heterophyllus Lam. } \\
\text { Moraceae }\end{array}$ & palamaram & $\begin{array}{l}\text { Seed, leaves, latex, } \\
\text { roots, and woods }\end{array}$ & $\begin{array}{l}\text { Seeds - relieve biliousness, aphrodisiac } \\
\text { Leaves - ulcers } \\
\text { Latex - promotes healing of abscesses, } \\
\text { snakebites, and glandular swelling } \\
\text { Root - skin diseases } \\
\text { Woods have sedative property }\end{array}$ \\
\hline 122. & $\begin{array}{l}\text { Alpinia galanga Sw. } \\
\text { Zingiberaceae }\end{array}$ & Aruttai & Whole plant & $\begin{array}{l}\text { Sore throat and hoarseness of the voice, bad } \\
\text { odor mouth, indigestion, and inflammations }\end{array}$ \\
\hline 123. & $\begin{array}{l}\text { Curculigo orchioides Gaertn. } \\
\text { Amaryllidaceae }\end{array}$ & Nilapanai kilangu & Rootstocks & $\begin{array}{l}\text { Skin diseases, asthma, bronchitis, jaundice, } \\
\text { diarrhea, dyspepsia, colic, vomiting, the dried } \\
\text { rhizome used as a tonic in the treatment of } \\
\text { decline in physical strength, sexual weakness, } \\
\text { leukorrhea, and menstrual disorders }\end{array}$ \\
\hline
\end{tabular}


Table 1: (Continued)

\begin{tabular}{|c|c|c|c|c|}
\hline S. No. & Botanical name/family & $\begin{array}{l}\text { Vernacular name/ } \\
\text { common name }\end{array}$ & Parts used & Therapeutic uses \\
\hline 124. & Dioscorea bulbifera L. Dioscoreaceae & Kaatu seerakavalli & Whole plant & $\begin{array}{l}\text { Ulcers, boils, wounds, sores, diabetes, dysentery, } \\
\text { and cancer }\end{array}$ \\
\hline 125. & $\begin{array}{l}\text { Asparagus racemosus Wild. } \\
\text { Liliaceae }\end{array}$ & Thaneervittan kilangu & Roots & $\begin{array}{l}\text { Galactagogue, diuretic, laxative, and } \\
\text { antidiarrhetic properties }\end{array}$ \\
\hline 126. & Gloriosa superba L. Liliaceae & Senkanthal & $\begin{array}{l}\text { Whole plant and } \\
\text { leaves }\end{array}$ & $\begin{array}{l}\text { Chronic ulcers, colic pain, gonorrhea, and pile } \\
\text { Leaves - asthma }\end{array}$ \\
\hline 128. & $\begin{array}{l}\text { Furcraea foetida (L.) Haw. } \\
\text { Liliaceae }\end{array}$ & Kattralai & Leaf paste & Useful in preparing shampoos \\
\hline 129. & $\begin{array}{l}\text { Scilla indica Bak. } \\
\text { Liliaceae }\end{array}$ & Kozhi vengayam & Whole plant & $\begin{array}{l}\text { Diuretic, deostruent, emetic, emmenagogue, } \\
\text { expectorant, cathartic, and anticancer agent }\end{array}$ \\
\hline 130. & $\begin{array}{l}\text { Spirodela polyrhiza (L.) Schleid. } \\
\text { Araceae }\end{array}$ & C.N - duck weed & Whole plant & $\begin{array}{l}\text { To treat inflammation, chronic rheumatism, and } \\
\text { rashes in skin, swellings, and eczema }\end{array}$ \\
\hline 132. & $\begin{array}{l}\text { Vetiveria zizanioides Nash. } \\
\text { Poaceae }\end{array}$ & Vettiver & Roots & Used as a refrigerant \\
\hline 133. & $\begin{array}{l}\text { Actiniopteris radiata (J. Koenig ex Sw.) } \\
\text { Link. } \\
\text { Actiniopteridaceae }\end{array}$ & Visirivalai & Whole plant & $\begin{array}{l}\text { Plant extract - to control blood pressure, } \\
\text { tuberculosis } \\
\text { Dried plant - cough }\end{array}$ \\
\hline 134. & $\begin{array}{l}\text { Hemionitis arifolia (Burm. f.) T. Moore. } \\
\text { Pteridaceae }\end{array}$ & Ithaya illai & Whole plant & $\begin{array}{l}\text { Juice of plant - cure burns and the plants } \\
\text { possess hypoglycemic and antidiabetic activities }\end{array}$ \\
\hline 135. & $\begin{array}{l}\text { Pteris tremula R.Br. } \\
\text { Pteridaceae }\end{array}$ & & Whole plant & $\begin{array}{l}\text { Stomach disorders, poisonous bites, } \\
\text { rheumatism, asthma, fever, and diabetes }\end{array}$ \\
\hline 136. & Adiantum caudatum L. Adiantaceae & Anai chuvadi & Whole plant & $\begin{array}{l}\text { Plant possesses analgesic, antinociceptive, anti- } \\
\text { implantation, and antimicrobial activities. To } \\
\text { treat diabetics, coughs, fever, migraine, and skin } \\
\text { diseases }\end{array}$ \\
\hline 137. & $\begin{array}{l}\text { Adiantum lunulatum Burm. fil. } \\
\text { Adiantaceae }\end{array}$ & Seruppadai & Whole plant & Cough, diarrhea, burns, and hoarseness \\
\hline
\end{tabular}

with binomial, local name, description of the plant, and uses. A survey of literature was made to find out the active principles of drug plants identified [22-24].

\section{RESULTS}

The present survey at Eratti hill Bargur range of reserve forest has documented a total of 289 angiospermic plants belonging to 66 families, six pteridophytic plants belonging to three families, one bryophyte thallus, and one type of foliose lichen. Of these 289 angiospermic plants, 273 dicotyledons and 16 monocotyledonous plants, from the dicotyledons 107 species were polypetalae plants, 126 were gamopetalae plants, and 56 were monochlamydeae plants, these were used to treat various ailments such as diabetes, eczema, fever, cold, cough, toothache, rheumatoid arthritis, liver disorders, cardiac disorders, microbial infections, headache, and blood pressure. The family-wise analysis showed that the families Asteraceae, Acanthaceae, and Euphorbiaceae held the dominant position by representing more number of species (17 species each), followed by Fabaceae (14 species), Convolvulaceae (13 species), Solanaceae (12 species), Amaranthaceae (11 species), Lamiaceae (11 species), Asclepiadaceae ( 9 species), Malvaceae ( 9 species), Mimosaceae ( 8 species), Apocynaceae (8 species), Capparidaceae (6 species), Tiliaceae, Sapindaceae, Caesalpiniaceae, Gentiaceae, Boraginaceae, Verbenaceae ( 5 species each), Liliaceae (4 species each), Rhamnaceae, Pedaliaceae, Loranthaceae (3 species each), Sterculiaceae, Vitaceae, Combretaceae, Myrtaceae, Passifloraceae (3 species each), Oxalidaceae, Simaroubaceae, Meliaceae, Anacardiaceae, Rosaceae, Aizoaceae, Chenopodiaceae, Aristolochiaceae, Santalaceae, Moraceae, Polygonaceae, and Dioscoreaceae (2 species each) and the remaining families such as Ranunculaceae, Annonaceae, Menispermaceae, Nymphaeaceae, Papaveraceae, Violaceae, Polygalaceae, Portulacaceae, Dipterocarpaceae, Zygophyllaceae, Celastraceae,
Droseraceae, Lythraceae, Onagraceae, Apiaceae, Caprifoliaceae, Plumbaginaceae, Myrsinaceae, Ebenaceae, Loganiaceae, Nyctaginaceae, Lauraceae, Ulmaceae, Orchidaceae, Zingiberaceae, Amaryllidaceae, Palmaceae, and Araceae were represented by a single species each. From the survey in the study, area represented the herbs were held the dominant position followed by the shrubs, trees and climber, four parasitic plants, and one epiphytic plant were reported (Table 1)

\section{DISCUSSION}

The observation of the present study at Eratti hill showed that traditional medicine plays an important role in the life of tribal communities. The medicinal plants used as a therapeutic agent of a paramount importance in addressing health problems of traditional communities and third world countries as well as industrialized societies [25]. Habit wise analysis represents the dominance of herbs followed by shrubs, trees, and climbers. These diverse habits indicate the richness of all habits. One epiphytic plant and four parasitic plants were reported. This is in line with the findings of Venkataswamy et al. [26], in Malasar tribals, Coimbatore district, (2010) and Arunachalam and Parimelazhgan [27], in Kadambur hills (2011). This study found that though whole and different parts of the medicinal plants were used as medicine, the most commonly used plant part was leaves. This is in agreement with the earlier findings of Ranganathan et al. [28], Bose et al. [29], Alagesaboopathi [30], Gritto et al. [31], and Sathyaraj et al. [32]. The family-wise analysis represents that the family Asteraceae, Acanthaceae, and Euphorbiaceae held the dominant position by exhibiting more number of species (17 species each). This is followed by Fabaceae (14 species) and Convolvulaceae (13 species). This is in consonance with the findings of Senthilkumar et al. [33], in Malayali tribes, Yercaud hills (2013). 
Western Ghats is one of the plant biodiversity hotspots of India. Agumbe region of Western Ghats is known for rich plant diversity and traditional medicinal practices. Raveesha and Nagabhushan [34] documented ethnomedicinal practices followed in this region to treat fungal infections and their scientific validation in vitro.

Vidyasagar and Pooja [35] selected the work deals with the studies on ethnomedicinal plants used by Rajgond Tribes of Haladkeri village in Bidar district, Karnataka, India. A total of 60 ethnomedicinal plants belonging to 37 families were recorded during the survey. The leaves were mostly used parts, followed by fruit, root, bark, flower, stem, and latex. These plants are being used to treat various ailments such as injuries, wounds, mouth ulcers, fever, diarrhea, ulcers, swelling, snakebite skin care, toothache, asthma, cough and cold diabetes, and cancer.

\section{CONCLUSION}

Medicinal plants in Eratti hill play a significant role in primary health care of the ethnic people. In the present study, 295 plants were documented and among these 289 plants were angiospermic plants, 6 were pteridophytic plants, one bryophytic thallus, and one type of foliose lichen. The plant species were used as a remedy for certain ailments. This study provides knowledge about herbal treatment of the ethnic people and subsequent pharmacognostical and pharmacological investigations should be made to confirm their mode of preparations.

\section{AUTHOR'S CONTRIBUTIONS}

Vijayashalini, P. carried out the study and was the charge of overall performance and planning. Abirami, P. has suggested the study and supervised the research work.

\section{CONFLICTS OF INTEREST}

The authors acknowledge that there are no conflicts of interest concerning this article publication.

\section{GRAPHICAL ABSTRACT}

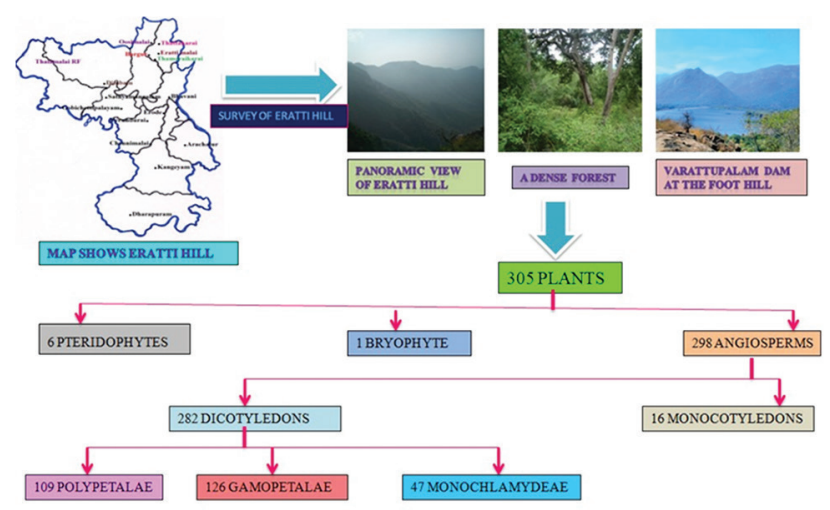

\section{REFERENCES}

1. Calixto JB. Twenty five years of research on medicinal plants in Latin America: A personal review. J Ethnopharmacol 2005;100:131-4

2. Bopana N, Saxena S. Asparagus racemosus-ethnopharmacological evaluation and conservation needs. J Ethnopharmacol 2007;110:115.

3. Amuthavalluvan V. Ethno medicinal practices and traditional healing system of Kattunayakan in Tamilnadu: An anthropological study. Int Mult Res J 2011;1:47-51.

4. Shanmugam S, Rajendran K, Suresh K. Traditional uses of medicinal plants among the rural people in Sivagangai district of Tamil Nadu, Southern India. Asian Pac J Trop Biomed 2012;5:429-34.

5. Purusothaman KG, Arun P, Jayarani JJ, Kumari RV. Screening of four Indian medicinal plants for in vitro antimycobacterial activity. Bioscan 2010;5:101-3.

6. Shiragave PD. Survey of medicinal plants used by local people of
Gadhinglaj tahsil of Maharashtra. J Global Biosci 2015;4:1795-803.

7. Sheng-Ji P. Ethnobotanical approaches of traditional medicine studies: Some experiences from Asia. Pharm Biol 2001;39 Suppl 1:74-9.

8. Samy PR, Ignachimuthu S. Antibacterial activity of some folklore medicinal plant used by tribals in Western Ghats of India. J Ethnopharmacol 2000;69:63-71.

9. Kunwar RM, Duwadee NP. Ecology and economy of NTFP's in Nepal: A case study from Dolpa and Jumla districts, Nepal. Botanica Orientalis 2003;3:89-97.

10. Heinrich M. Ethnobotany and its role in drug development. Phytother Res 2000;14:479-88.

11. Carney JR, Krenisky JM, Williamson RT, Luo J, Carlson TJ, Hsu VL, et al. Maprouneacin, a new daphnane diterpenoid with potent antihyperglycemic activity from maprounea africana. J Nat Prod 1999;62:345-7.

12. Jain SK, Rao RR. Hand Book of Field and Herbarium Methods. New Delhi: Today and Tomorrow Publishers; 1977.

13. Gamble JS, Fischer CE. The Flora of the Presidency of Madras. Vol. I, II and III. London: Adlard and Son Ltd.; 1915-1936.

14. Matthew KM. Materials for a Flora of the Tamil Nadu Carnatic. Vol. I. Tamil Nadu: Diocesan Press; 1981.

15. Matthew KM. Illustrations on the Flora of the Tamil Nadu Carnatic. Vol. II. Tamil Nadu: Diocesan Press; 1982.

16. Matthew KM. The Flora of the Tamil Nadu Carnatic. Vol- III. Part- I, II \& III. Thiruchirapalli, India: The Rapinet herbarium, ST.Joseph's College; 1983.

17. Matthew KM. Further Illustrations on The Flora of the Tamil Nadu Carnatic. Vol. IV. New Delhi: Oxford and IBH Publishing Co.Pvt.Ltd.; 1988.

18. Matthew KM. An Excursion Flora of Central Tamil Nadu, India. New Delhi: Oxford and IBH Publishing Co.Pvt.Ltd; 1991.

19. Nair NC, Henry AN. Flora of Tamil Nadu, India. Ser. I, Anaysis. Vol. I. Coimbatore: Botanical survey of India; 1983.

20. Henry AN, Chitra V, Balakrishnan NP. Flora of Tamil Nadu, India. Ser. I, Analysis. Vol. III. Coimbatore: Botanical survey of India; 1989.

21. Henry AN, Kumari GR, Chitra V. Flora of Tamil Nadu, India. Ser. I, Analysis. Vol. II. Coimbatore: Botanical survey of India; 1987.

22. Chopra RN, Nayar SL, Chopra IC. Glossary of Indian Medicinal Plants. New Delhi: CSIR; 1956.

23. Jain SK. Dictionary of Indian Folk Medicines and Ethno Botany. New Delhi: Deep publications; 1991.

24. Asolkar LV, Kakkar KK, Chakre OJ Second Supplement to Glossary of Indian Medicinal Plants with Active Principle. Part. I (A-K). New Delhi: CSIR; 1992.

25. Cano JH, Volpato GJ. Herbal mixtures in the traditional medicine of eastern Cuba. J Ethnopharmcol 2004;90:293-316.

26. Venkataswamy R, Mubarack HM, Doss A, Ravi TK, Sukumar M. Ethnobotanical study of medicinal plants used by Malasar tribals in Coimbatore district of Tamil Nadu (South India). Asian J Exp Biol Sci 2010;1:387-92.

27. Arunachalam K, Parimelazhagan T. Ethnomedicinal observations among Hooralis tribes in Kadambur hills (Kal Kadambur) Erode District, Tamil Nadu. Glo J Pharma 2011;5:117-21.

28. Ranganathan R, Vijayalakshmi R, Parameswari P. Ethnomedicinal survey of Jawadhu hills in Tamil Nadu. Asian J Pharm Clin Res 2012;5:45-9.

29. Boss NM, Aron S, Mehalingam P. An ethnobotanical study of medicinal plants used by Paliyars aboriginal community in Virudhunagar District, Tamil Nadu, India. I. J Trad Know 2014;13:613-8.

30. Alagesaboopathi C. Medicinal plants used for the treatment of liver diseases by Malayali tribes in Shervaroy hills, Salem District, Tamil Nadu, India. World J Pharm Res 2015;4:816-28.

31. Gritto MJ, Nanadagopalan V, Doss V. Ethnobotanical survey of medicinal plants used by Traditional healers in Shobanapuram Village of Pachamalaihill, TamilNadu. Adv Appl Sci Res 2015;6:157-64.

32. Sathyaraj R, Sarvalingam AA, Balachandran A, Reddy RK. Diversity of ethnomedicinal plants in Bodamalai hills, Eastern Ghats, Namakkal District, Tamil Nadu. J Plant Sci 2015;3:77-84.

33. Senthilkumar K, Aravindhan V, Rajendran A. Ethnobotanical survey of medicinal plants used by Malayali tribes in Yercaud hills of Eastern Ghats, India. J Nat Remedies 2013;13:118-32.

34. Nagabhushan RK. Ethnobotanical survey and scientific validation of medicinal plants used in the treatment of fungal infections in Agumbe Region of Western Ghats, India. Int J Pharm Pharm Sci 2015;7:273-7.

35. Vidyasagar GM, Pooja S. Ethnomedicinal plants used by Rajgond tribes of Haladkeri Village in Bidar District, Karnataka, India. Int J Pharm Pharm Sci 2015;7:216-20. 